\title{
Verfassungslos: Verfassungs- und staatsrechtliche Bedenken anlässlich des Bundesgerichts- entscheides zur Bündner Spracheninitiative
}

Thomas Burri *

Der jüngste Bundesgerichtsentscheid zur Bündner Spracheninitiative hat Schwächen. Doch diese liegen nicht etwa in der bundesgerichtlichen Argumentation. Sie finden ihre Ursache vielmehr in der Verfassung selbst. Der Entscheid und die gesamte Sachlage offenbaren, dass der dem schweizerischen Bundesstaat angeblich immanente Schutz sprachlicher Minderheiten sowie die Toleranz und die Rücksicht, auf welche die Verfassung baut, nicht ausreichen, um Selbstbestimmung, Freiraum und Schutz der sprachlichen Minderheiten vor Unterdrückung zu gewährleisten.

I. Einleitung: Zwei Fragen

II. Das internationale Minderheitenrecht ist schwach und bietet keinen Schutz. 150

III. Das Bundesgericht hat gesprochen und liegt richtig 151

IV. Verfassungslos - oder: die Schönwetterverfassung 151

V. Die Tradition der Schweiz - und des Völkerbundes..................................... 153

VI. Der Romanenstaat: Er ist zurück ............................................................ 154

VII. Übertriebene Eigenstaatlichkeit der Kantone im Bundesstaat ....................... 155

VIII. Schluss 156

Zitiervorschlag: Thomas Burri, Verfassungslos: Verfassungs- und staatsrechtliche Bedenken anlässlich des Bundesgerichtsentscheides zur Bündner Spracheninitiative, in: sui-generis 2017, S. 148

URL: $\quad$ sui-generis.ch/40

DOI: $\quad$ https://doi.org/10.21257/sg.40

* Prof. Dr. Thomas Burri, Assistenzprofessor für Völkerrecht und Europarecht an der Universität St.Gallen (HSG); Dr. iur. (Zürich), LL.M. (College of Europe, Brügge), lic.iur. (Basel), Rechtsanwalt in Zürich, PD (venia legendi für Völkerrecht, Europarecht und Verfassungsrecht). Thomas Burri ist Stiftungsrat bei Convivenza - Internationales Zentrum für Minderheiten. Kontakt: <thomas.burri@unisg.ch>, <www.thomas-burri.com>. 


\section{Einleitung: Zwei Fragen}

1 Erste Frage: Darf die Mehrheit sich weigern, die Sprache der Minderheit zu lernen?

2 Zweite Frage: Darf die Mehrheit die Minderheit zwingen, die Sprache der Mehrheit als einzige Fremdsprache zu lernen?

3 Die Fragen klingen kompliziert. Doch man sollte sich die Zeit nehmen, sie zu verstehen, denn die Antworten darauf sind essenziell für das Staatsverständnis, ja gar für die Zukunft, der Schweiz. Das Bundesgericht hat eben in einem neuen Entscheid beide Fragen bejaht. ${ }^{1}$ Der Entscheid fiel anlässlich einer Beschwerde gegen eine Gesetzesinitiative im Kanton Graubünden, die vorsah, dass in den Primarschulen im ganzen dreisprachigen Kanton bloss noch eine Fremdsprache obligatorisch gelehrt werden darf, statt wie bisher deren zwei. ${ }^{2}$ Im Falle der Annahme der Initiative würde dies für die deutschsprachigen Gebiete in Graubünden voraussichtlich bedeuten, dass auf Primarstufe einzig Englisch obligatorisch als Fremdsprache unterrichtet werden darf (erste Frage oben), während in den italienisch- und rätoromanischsprachigen Gebieten ausschliesslich Deutsch gelehrt werden darf (zweite Frage).

\footnotetext{
Urteil des Bundesgerichts 1C_267/2016 vom 3. Mai 2017.

2 Der Text der Initiative lautet: «Das Gesetz für die Volksschulen des Kantons Graubünden ist so abzuändern und auszugestalten, dass in der Primarschule für den Fremdsprachenunterricht im ganzen Kanton folgende Regel gilt: In der Primarschule ist nur eine Fremdsprache obligatorisch, je nach Sprachregion ist dies Deutsch oder Englisch.» (Siehe Paragraph A in Urteil des Bundesgerichts 1C_267/2016 vom 3. Mai 2017).
}

4 Werden die beiden Fragen vom konkreten Anlass losgelöst betrachtet, lautet die intuitive, sicher mehrheitsfähige Antwort auf die erste Frage: Es ist zwar nicht schön, wenn die Mehrheit sich weigert, die Sprache der Minderheit zu lernen und dies wird auch den Zusammenhalt der Gesellschaft und das Verhältnis zwischen Mehrheit und Minderheit nicht positiv beeinflussen, doch die Mehrheit muss die Sprache der Minderheit nicht zwingend lernen. Dies gilt insbesondere nicht, wenn Kenntnisse einer anderen Fremdsprache unabdingbar geworden sind. Während das Bejahen der ersten Frage lediglich gewisse Bedenken hervorruft, löst das blosse Stellen der zweiten Frage tiefes Unbehagen aus. Die Minderheit wird durch die Pflicht, ausschliesslich die Sprache der Mehrheit als Fremdsprache zu lernen, stark eingeschränkt. Das Bedürfnis der Minderheit, ihre kulturelle Entwicklung selbst zu bestimmen, wird negiert und dies im Bereich der Sprache, der für Minderheiten essenziell ist. Mit anderen Worten geht damit ein gewisses Mass an Unterdrückung der Minderheit einher. Die Intuition legt daher eine negative Antwort auf die zweite Frage nahe oder im besten Falle sollte die Minderheit selbst darüber entscheiden können. Diese Indikation fällt besonders deutlich aus angesichts des Zusammenhanges zwischen den beiden Antworten: Wenn die Mehrheit selbst die Sprache der Minderheit vernachlässigt, sollte sie doch die Minderheit nicht zwingen dürfen, ausschliesslich ihre Sprache zu lernen. Die Initution rät also: Die Antworten sollten erstens «Ja» und zweitens «Nein» lauten, bzw. wenn schon erstens «Ja», dann mindestens auch zweitens «Nein». 


\section{Das internationale Minderheiten- recht ist schwach und bietet keinen Schutz}

5 In den obigen, intuitiven Antworten widerspiegelt sich der heutige internationale Minderheitenschutz. Dieser erlegt dem Staat - und damit der jeweiligen Mehrheit - relativ beschränkte positive Pflichten auf. Internationalrechtliche Vorgaben des Minderheitenschutzes zwingen die Mehrheit nicht, die Sprache der jeweiligen Minderheit zu lernen. Hingegen wird der mehrheitliche Staat verpflichtet, der jeweiligen Minderheit einen Raum zur Entfaltung sowie Autonomie und Selbstbestimmung zu gewähren, etwa in sprachlichen und kulturellen Aspekten.

6 Doch dieser internationale Minderheitenschutz ist schwach. Er besteht in einem einzigen verbindlichen Artikel, nämlich Artikel 27 des Bürgerrechtspaktes 3 , und einer Anhäufung von grösstenteils unverbindlichen Erklärungen 4 oder schwammigen und zahnlosen, weil nicht unmittelbar wirksamen, Abkommen.5

Internationaler Pakt vom 16. Dezember 1966 über bürgerliche und politische Rechte (SR 0.103.2, UNO-Pakt II). Artikel 27 lautet: «In Staaten mit ethnischen, religiösen oder sprachlichen Minderheiten darf Angehörigen solcher Minderheiten nicht das Recht vorenthalten werden, gemeinsam mit anderen Angehörigen ihrer Gruppe ihr eigenes kulturelles Leben zu pflegen, ihre eigene Religion zu bekennen und auszuüben oder sich ihrer eigenen Sprache zu bedienen.»

4 Erklärung über die Rechte von Personen, die nationalen oder ethnischen, religiösen und sprachlichen Minderheiten angehören, UN Generalversammlung, (A/RES/47/135, GAOR $47^{\text {th }}$ session sup. 49 vol 1, 199 ) vom 18. Dezember 1992; Erklärung über die Rechte der eingeborenen Völker, UN Generalversammlung (A/RES/61/295, GAOR 61th session suppl. 49 vol. 3, 15) vom 13. September 2007.

5 Rahmenübereinkommen zum Schutz nationaler Minderheiten vom 1. Februar 1995 (SR 0.441.1) (Europarat CETS no. 157); Europäische Charta der Regional- oder Minderheitensprachen vom
Das Selbstbestimmungsrecht der Völker wiederum, das im gemeinsamen Artikel 1 des internationalen Bürger-, bzw. Sozialrechtspaktes niedergelegt ist, ${ }^{6}$ hat nicht die Kraft, Minderheiten ausserhalb von Extremsituationen effektiv zu schützen. So bleiben denn Minderheiten international gerade in kulturellen Belangen weitgehend schutzlos, auch in etablierten Demokratien und mächtigen Staaten. Dies gilt insbesondere, wenn ein Vorstoss nicht auf die Eigensprache einer Minderheit oder deren Kenntnisse der Mehrheitssprache zielt, sondern wie hier auf die erweiterten Sprachkenntnisse in der heutigen «Lingua franca», die für den beruflichen und kompetitiven Erfolg und somit die freie Entfaltung von Minderheitenangehörigen in höchstem Masse relevant sind. In dieser Hinsicht reduziert sich der ohnehin kaum griffige internationale Minderheitenschutz auf den vagen Schutz des «eigenen kulturellen Lebens» (Artikel 27), da die eigentliche «eigene Sprache» (Artikel 27) der Minderheit nicht betroffen ist.

7 Doch wen wundert es, dass der internationale Minderheitenschutz schwach ist? Die jeweiligen Mehrheiten, die in aller Regel die Staaten führen und das Völkerrecht prägen, betreiben doch den Minderheitenschutz. Oft halten sie ihn bewusst klein, betreiben ihn einseitig und in paternalistischer Weise und unterwandern ihn so. Daniel Thürer weist zudem darauf hin, dass zwischen den Staaten oft stille Solidarität herrscht: "Indeed, States sometimes behave like ,cartels' seeking to protect their power and shielding it off from external interfe-

5. November 1992 (SR 0.441.2) (Europarat CETS no. 148).

6 Siehe Art. 1 UNO-Pakt II. 
rence.»7 Den Minderheiten bleibt dann nur der Rückgriff auf die allgemeinen menschenrechtlichen Garantien, die abgesehen von Extremsituationen ungeeignet sind, die besonderen Anliegen von Minderheiten positiv zur Geltung zu bringen - nicht zuletzt da die Angehörigen der Mehrheit - zu recht - auch Menschenrechte haben, gegen die es stets abzuwägen gilt.

\section{Das Bundesgericht hat gesprochen und liegt richtig}

Was ist aber nun mit dem doppelten «Ja» des Bundesgerichtes auf die zwei eingangs gestellten Fragen? Hätten die Beschwerdeführenden vor Bundesgericht lediglich einer besseren anwaltlichen Vertretung bedurft? Wohl kaum. Auch wenn die positive Antwort des Bundesgerichtes auf die zweite Frage kontraintuitiv erscheint, ist sie nicht deswegen falsch oder gar widerrechtlich. Es ist schlicht die Antwort, die sich aus einer ausgewogenen, wenn auch positivrechtlich geprägten Lektüre des Verfassungstextes ergibt. Das Ergebnis drängt sich sogar geradezu auf, wenn man die von der Bundesverfassung betonte Eigenstaatlichkeit der Kantone ernst nimmt. Wo sonst hätten die Kantone überhaupt noch Kompetenzen, wenn nicht in der Gestaltung der Eigensprache und des Schulunterrichts? Die Zersplitterung in 26 staatstragende Entitäten bzw. deren Nichthomogenisierung - ist ein gewolltes Element der Föderation Schweiz. Schefer/Rüegger sprechen in dieser Hinsicht von der «Vielfalt der Kantone als Leitgedanke für den inneren

\footnotetext{
Daniel Thürer, «Minorities and Majorities: Managing Diversity - a fresh look at an old problem», 15 SZIER (5) (2005), S. 659 ff., S. 659.
}

Zusammenhalt des Bundesstaates und den Umgang mit der kulturellen Vielfalt» 8 . Die Nachteile, die daraus folgen, können nicht durch eine progressive Interpretation des Verfassungstextes herausgelesen, die Balance der Kompetenzen nicht leise verschoben werden. 9

9 Der Text der Schweizer Verfassung ist, was er ist. Das Bundesgericht hat ihn angewendet und hat dabei dem Respekt vor den Kantonen als staatsbildenden Einheiten die Treue gehalten. Progressive, aktivistische Schritte, die den Verfassungstext gedehnt hätten und die nötig gewesen wären, um die Bündner Spracheninitiative als verfassungswidrig zu befinden, wären in der Schweiz Sache des Gesetz- oder Verfassungsgebers, nicht der Judikative. Die Bundesrichterschaft hat also ihre Pflicht getan, alles lief rechtens und die Sache ist damit erledigt. Der Gang nach Strassburg erscheint im Übrigen auch aussichtslos.

\section{Verfassungslos - oder: die Schön- wetterverfassung}

10 Dennoch bleibt ein ungutes Gefühl, ein Unbehagen. Der Entscheid widerspricht doch der Intuition, wie eingangs angedeutet wurde. Da das Bundesgericht die

$8 \longdiv { \text { Markus Schefer / Vanessa Rüegger, «Die Pflicht } }$ der Kantone zur Koordination des Sprachenunterrichts (Art. 62 BV)» recht (2015), S. 226 ff., S. 228.

9 Siehe auch Andreas Glaser, «Die Kompetenz der Kantone zur Regelung des Fremdsprachenunterrichts in der Primarschule» Schweizerisches Zentralblatt für Staats- und Verwaltungsrecht (117) (2016), S. 139 ff., S. 144: «Kantone, die einen Beitritt zum HarmoSKonkordat ausdrücklich abgelehnt haben, können nicht durch die Hintertür einer offen formulierten Verfassungsbestimmung den identischen Pflichten unterworfen werden wie die Kantone die dem HarmoS-Konkordat beigetreten sind.» Das Bundesgericht teilte diese Ansicht in seinem Urteil 1C_267/2016 vom 3. Mai 2017, Erw. 7.2. 
Verfassung aber lediglich anwandte, muss der Missstand in der Verfassung selbst angelegt sein. Sicher, die Schweizer Verfassung ist zweifelsohne von herausragender Qualität - aber nicht in Sachen Minderheitenschutz. Beim Minderheitenschutz ist sie schwach und bei den für die Minderheiten besonders wichtigen Sprachkompetenzen verstrickt sie sich in einem Kompetenzgewirr. Hat der Bund nun die Sprachkompetenz oder nicht? Nein, aber ja. Was in diesen Verfassungstext an Minderheitenschutz hineingelesen werden kann, wurde bereits hineingelesen. ${ }^{10}$ Der Missstand liegt aber genau darin: Der Minderheitenschutz in der Schweiz muss, um gefunden zu werden, in den Verfassungstext hineingelesen werden. Er steht schlicht und einfach nicht drin. Die Minderheiten sind in der Schweiz verfassungslos.

Die Verfassung beschränkt sich auf indirekte Hinweise mittels der Sprachenfreiheit, des Diskriminierungsschutzes oder der Sprachkompetenzen. Darüber hinaus vertraut die Schweizer Verfassung darauf, dass der Schutz von Minderheiten dem bundesstaatlichen Aufbau der Schweiz immanent ist. Aus einer Kombination von Gemeinden, Kantonen und

10 Adriano Previtali, «Nur eine Fremdsprache in der Primarschule? Ein Rechtsgutachten (Übersetzung)» Zeitschrift für Gesetzgebung und Rechtsprechung in Graubünden (2) (2014), S. 93 ff.; Bernhard Ehrenzeller, Gutachten zur Frage der Gültigkeit der kantonalen Volksinitiative «Nur eine Fremdsprache in der Primarschule»; Schefer / Rüegger (Fn. 8); Glaser (Fn. 9); Siehe aber auch Bernhard Waldmann, "Besteht eine Bundeskompetenz zur Regelung des Fremdsprachenunterrichts?» Newsletter IFF (1) (2015), S. $17 \mathrm{ff}$. $\mathrm{Zu}$ den kantonalen Fremdspracheninitiativen, siehe Andreas Lienhard / Kurt Nuspliger, «Kantonale Fremdspracheninitiativen im Kontext übergeordneten Bundesrechts» Schweizerisches Zentralblatt für Staats- und Verwaltungsrecht (117) (2016), S. $115 \mathrm{ff}$.
Bund mit ihren jeweils eigenen Rechtsetzungskompetenzen soll sich der Minderheitenschutz gleichsam organisch ergeben, ohne dass er explizit thematisiert werden muss. Es bleibt damit ein weites Feld für die Politik, die sich auf allen Ebenen in Wohlwollen, Toleranz und Rücksicht übt und oft ad hoc bei konkreten Entscheiden den Minderheiten und ihren Angehörigen ein überproportionales Gewicht gewährt.

12 Dies sind jedoch die Züge einer Schönwetterverfassung. Die Qualität und Stärke einer Verfassung misst sich auch an der Zufriedenheit und dem Wohlwollen aller und daran, dass die ausreichend vorhandenen Ressourcen auf alle Bedürfnisse gerecht verteilt werden. Doch die Qualität und Stärke einer Verfassung tritt erst zu Tage, ${ }^{11}$ wenn Druck aufkommt, wenn ein Mehrheitsführer oppressiv $\mathrm{zu}$ regieren beginnt und gegen Minderheiten politisiert, um die eigene Basis zu befeuern und seine Macht auszuweiten - ein Test, der derzeit in den USA und in der Türkei im Gange ist. Erst in diesem Fall zeigt sich, ob eine Verfassung widerstandsfähig ist und Minderheiten effektiv zu schützen vermag. Dann aber erweist sich expliziter Minderheitenschutz mit garantierten Vertretungen, geschütztem Eigenraum, qualifizierten Mehrheitserfordernissen, übergreifender, unparteiischer Aufsicht, funktionaler Anknüpfung und wirkungsorientiertem Diskriminierungsschutz als wirkungsvoller als das blosse Vertrauen auf einen systemimmanenten Schutz.

11 Nur der Klarheit halber: Es wird hier keineswegs einer Verfassungskonzeption im Sinne Schmitts das Wort geredet - im Gegenteil (siehe Carl Schmitt, Politische Theologie - Vier Kapitel zur Lehre von der Souveränität, 2. A., München 1934). 


\section{Die Tradition der Schweiz - und des Völkerbundes}

13 Solche Schutzmechanismen, wird man entgegnen, entsprechen nicht der Tradition der Schweiz. Doch stehen die Bündner Spracheninitiative oder die Volksinitiativen auf nationaler Ebene über das Völkerrecht (sog. «Selbstbestimmungsinitiative» - man beachte die manipulative Verdrehung des Selbstbestimmungsrechts), über die angebliche Masseneinwanderung, die Ausschaffung, das Minarettverbot, oder die Verwahrung ebensowenig in einer Schweizer Tradition der Toleranz und des Respekts. Sie alle schaffen eben solche Drucksituationen, in denen systemimmanentes Wohlwollen zugunsten von Minderheiten (und etwaigen weiteren Personengruppen) keinen ausreichenden Schutz bietet. Sie entlarven auch die weit verbreitete Vorstellung, dass Drucksituationen in Bezug auf Minderheiten bloss anderswo aber nicht in der Schweiz auftreten, als Irrglauben. Die Schweiz ist nicht immun. Im Gegenteil - ihre allgemein flexibel gehaltene und in Bezug auf Minderheiten schwache Verfassung ist in höherem Masse anfällig für Manipulationen als etwa in den Nachbarländern, wo die Verfassungen stabiler und besser geschützt sind. Entsprechend knarrt und kracht es in jüngster Zeit im Schweizer Verfassungsgebälk.

Es entspricht auch nicht den Tatsachen, dass man in der Schweiz Minderheiten wie den Rätoromanen oder Italienischsprachigen im Graubünden so weit entgegenkommt wie überhaupt möglich von den Roma dort und anderswo ganz zu schweigen. Wie effektiver Minderheitenschutz funktionieren kann, hatte schon der Völkerbund gezeigt. Damals wurde der Minderheitenschutz den lokalen Verhältnissen und Machtspielen entzogen, in internationalen Abkommen verankert und unter internationale Aufsicht gestellt. So wurden Minderheitenstreitigkeiten wiederholt im Rat des Völkerbundes, der die Rolle des Garanten der Minderheitenrechte ausübte, verhandelt - etwa in Bezug auf die freie Stadt Danzig, die Ålandinseln oder Oberschlesien. ${ }^{12}$ Der Völkerbundsrat bezog in Minderheitenfragen auch den Ständigen Internationalen Gerichtshof regelmässig mit ein, gerade auch in heiklen Fragen des Minderheitenschulwesens. ${ }^{13}$

$12 \mathrm{Zu}$ den Åland Inseln, siehe: Report of the International Commission of Jurists Entrusted by the Council of the League of Nations with the Task of Giving an Advisory Opinion upon the Legal Aspects of the Aaland Islands Question, International Commission of Jurists, League of Nations Official Journal, Special Supplement No. 3, October 1920; The Åland Island Question: Report Submitted to the Council of the League of Nations by the Commission of Rapporteurs, Commission of Rapporteurs, League of Nations Council Doc. B7/21/68/106 VII, April 1921. Siehe auch Oliver Diggelmann, «The Aaland Case and the Sociological Approach to International Law», 18 EJIL (1) (2007), S. 135 ff. Zu Danzig und Oberschlesien siehe die Fälle in der folgenden Fussnote.

13 Siehe dazu die folgenden, teilweise bahnbrechenden Entscheidungen: Permanent Court of International Justice Bo6, advisory opinion no. 6 of 10 September 1923 (German Settlers in Poland) ; Permanent Court of International Justice, 1923 P.C.I.J. of 15 September 1923 (Acquisition of Polish Nationality); Permanent Court of International Justice B11, advisory opinion no. 11 of 15 September 1923 (Polish Postal Service in Danzig); Permanent Court of International Justice A06, judgment no. 6 of 25 August 1925 (Certain German Interests in Polish Upper Silesia); Permanent Court of International Justice A07, judgment no. 7 of 25 May 1926 (Certain German Interests in Polish Upper Silesia (The merits)); Permanent Court of International Justice A15, judgment no. 12 of 26 April 1928 (Rights of Minorities in Upper Silesia (Minority Schools)); Permanent Court of International Justice B15, advisory opinion no. 15 of 3 March 1928 (Jurisdiction of the Courts of Danzig); Permanent Court of International Justice, The Greco-Bulga- 
15 Wie nötig wäre doch ein solch neutraler Arbiter, der sich auf solide verankerte Minderheitengarantien berufen könnte, nun für die Schweiz insgesamt, aber auch für die Kantone und insbesondere das Graubünden. Eine solche Instanz müsste für die Kantone wirken und sich auf solide Verfassungsbestimmungen verlassen können. Für Bundesangelegenheiten wäre eine internationale Instanz vorzusehen, die reale Zuständigkeiten hätte, und nicht nur eine Empfängerin wirkungsloser und unverbindlicher Berichte wäre. Dass dies politisch nicht mehrheitsfähig ist, liegt in der Natur der Sache. Dies zeigt sich schon alleine daran, dass der Europäische Gerichtshof für Menschenrechte das Rahmenübereinkommen des Europarates zum Schutz nationaler Minderheiten - ein Rahmenübereinkommen! - nicht überwachen darf. Gerade weil derartiger Minderheitenschutz nicht mehrheitsfähig ist, ist er unbedingt notwendig.

rian «Communities», B17, advisory opinion no. 17; Permanent Court of International Justice B18, advisory opinion no. 18 of 31 July 1930 (Free City of Danzig and International Labour Organization); Permanent Court of International Justice, Access to German Minority Schools in Upper Silesia, A/B 40, advisory opinion; Permanent Court of International Justice A/B 43, advisory opinion of 15 May 1931 (Polish War Vessels in the Port of Danzig); Permanent Court of International Justice A/B 44, advisory opinion of 4 February 1932 (Treatment of Polish Nationals in Danzig); Permanent Court of International Justice A/B 47 of 24 June 1932 (Interpretation of the Statute of the Memel Territory (Preliminary Objection)); Permanent Court of International Justice A/B 49, of 11 August 1932 (Interpretation of the Statute of the Memel Territory); Permanent Court of International Justice A/B 64 of 6 April 1935 (Minority Schools in Albania); Permanent Court of International Justice A/B 65 of 4 December 1935 (Consistency of Certain Danzig Legislative Decrees With the Constitution of the Free City).

\section{Der Romanenstaat: Er ist zurück}

16 Der Schweizer Verfassung wohnt, wohl gemerkt, das Potenzial zum effektiven Minderheitenschutz inne. Doch wie es scheint bedarf es zu dessen Aktivierung grösserer Kraft, Mutes, Einsatzes und Extrovertiertheit als auf Seiten der Minderheiten (und natürlich der Mehrheit) vorhanden sind. Die Idee des Romanenstaates $^{14}$ war und ist durchaus ernst gemeint. Ein Romanenstaat - der, nur um Missverständnissen vorzubeugen, eigentlich ein Romanenkanton ist - würde mit Sicherheit effektiveren Freiraum, Schutz und Selbstbestimmung bieten, als es die gegenwärtige Bundesverfassung bewirkt, insbesondere in Bezug auf das Schulwesen. ${ }^{15}$ Dies dürfte spätestens seit dem jüngsten Bundesgerichtsentscheid klar sein. Der Romanenstaat ist auch eher als Generationenprojekt zu denken, denn als Generationenkonflikt - auch wenn es nicht überrascht, dass er als Letzteres porträtiert wird. Den Misserfolg einer langen Reihe versuchter Projekte einer ganzen Generation gibt diese nicht leichthin zu. Jede grundlegende Veränderung sieht sich zudem zunächst tief eingegrabenen Interessen gegenüber. Schliesslich ist ein Romanenstaat (oder auch ein Romastaat!) zwar ein kompliziertes und langfristiges, aber auch ein machbares Projekt - zumindest mach-

14 Thomas Burri, «Der Romanenstaat - Versuch einer verfassungsrechtlichen Lösung der Rätoromanenfrage», in Corsin Bisaz / Andreas Glaser (Hrsg.), Rätoromanische Sprache und direkte Demokratie, Zürich 2015, S. 125-131; siehe auch Thomas Burri, «Der Romanenstaat», NZZ vom 6. August 2014, S. 17.

15 Ein Romanenstaat würde ebenfalls die Befreiung der rätoromanischen Sprache aus den Fängen der Kantone und des Territorialprinzips bewirken. Dies fordert überzeugend Romedi Arquint, Plädoyer für eine gelebte Mehrsprachigkeit. Die Sprachen im Räderwerk der Politik, Zürich 2014. 
barer, sowohl in rechtlicher wie faktischer Hinsicht, als die Staaten Katalonien, Kosovo, und Kurdistan.

\section{VII. Übertriebene Eigenstaatlichkeit der Kantone im Bundesstaat}

17 Effektiver, übergreifender Minderheitenschutz wie er oben dargestellt wurde, wäre mit der Eigenstaatlichkeit der Kantone durchaus vereinbar. Doch selbst wenn nicht, darf deren Eigenstaatlichkeit nicht dazu dienen, Unterdrückung abzuschirmen. Föderation ist keine Rechtfertigung für Oppression. Der Fall vor Bundesgericht hat im Übrigen exemplarisch die sonderbaren Blüten gezeigt, die ein zwar wohlgemeinter, doch letztlich übertriebener Föderalismus und eine Überbetonung der kantonalen Eigenstaatlichkeit treiben. ${ }^{16}$

Die Verfassung verweist in Artikel 62 Absatz 4 die Harmonisierung des Schulwesens auf den «Koordinationsweg» und sieht in bestimmter Hinsicht eine Auffangkompetenz des Bundes vor, falls die Koordination nicht zustande kommt. Damit wird die Harmonisierung zunächst von den Bundesinstitutionen ferngehalten, obwohl diese eigentlich genau dafür geeignet wären. ${ }^{17}$ Das eigentlich dahinterstehende Anliegen, nämlich

$16 \overline{\text { Für die Details bezüglich des Konkordatswesens }}$ siehe Giovanni Biaggini, «Schulkoordination in der Schweiz: Der steinige Weg des «KonkordatsFöderalismus»» Jahrbuch des Föderalismus (2009), S. 380 ff. und Thomas Gächter, «Welche Gestaltungsspielräume verbleiben den Kantonen im Rahmen des HarmoS-Konkordats?», in Andreas Auer (Hrsg.), Herausforderung HarmoS: Bildungspolitik, Föderalismus und Demokratie auf dem Prüfstein, Zürich 2010, S. 59 ff.

17 Schefer / Rüegger (Fn. 8), S. 226, erwähnen die «stetige [...] Ausweitung der Kompetenzen des Bundes auf Kosten der Kantone seit der Gründung des Bundesstaates». keinem Kanton eine Harmonisierung aufzudrängen, der er nicht zustimmen kann, und damit die Eigenständigkeit der Kantone zu wahren, relativiert Artikel 62 Absatz 4 sogleich. Die sich sperrenden Kantone sollen sanktioniert werden können, indem die Kompetenz zumindest teilweise auf den Bund übergeht, wo mit Mehrheitsentscheidungen operiert wird und so der Wille eines Grossteils der Kantone ohne Weiteres übergangen werden kann.

19 Wie kurios und untauglich dieser Ansatz ist, zeigt sich in Fällen wie dem vorliegenden, in denen einige wenige Kantone, oder sogar ein einzelner, aus der Koordination ausreissen - und dies womöglich mit gutem Grund, etwa der einzigartigen Dreisprachigkeit. ${ }^{18}$ Soll nun der Bund das Schulwesen (teil)harmonisieren, nachdem die allermeisten Kantone sich einig sind und nur einzelne ausscheren? Eine Antwort ist schwierig zu geben und mit Unsicherheiten behaftet. Im Nachhinein kann man man jedoch zumindest sagen, dass es sinnvoller und rechtlich sauberer gewesen wäre, die Harmonisierung gleich auf Bundesebene zu verorten, die Ausübung der Kompetenz aber einem Einstimmigkeitserfordernis zu unterwerfen, auch wenn ein solches nicht den üblichen Gesetzgebungsprozessen der Verfassung entspricht. Für den (voraussehbaren) Fall, dass die Kompetenz dann nicht ausgeübt würde, wäre sodann eine differenzierte Integration vorzusehen gewesen. Die willigen Kantone hätten diese innerhalb der Bundesinstitutionen vorantreiben können. Das Einziehen einer zusätzlichen Zwischenschicht zwi-

18 Für die Entstehung des HarmoS-Konkordates mit allen Details, siehe Schefer / Rüegger (Fn. 8), S. 227. 
schen Kantone und Bund im Schulwesen, die in ihrer zwielichtigen Existenz rechtlich komplexe Fragen aufwirft, hätte sich so erübrigt. Der Bund wäre gar nicht erst in die heikle Lage gekommen, eine Kompetenz gegen und zulasten eines oder weniger Kantone ausüben zu müssen. Die Bundeskompetenz wäre ohne den unschönen Sanktionscharakter ausgekommen. Die fragwürdige Interpretation, welche Ausreisser in die Wirkung der Koordination miteinbezieht, wäre klarerweise unzulässig gewesen. Das Schulwesen wäre nicht künstlich von den bewährten Bundesinstitutionen ferngehalten worden, nur um eine falsche Eigenständigkeit der Kantone zu zelebrieren. Es würde auch klarer zu Tage treten, dass die Ziele der Bildungsverfassung Qualität und Durchlässigkeit des Bildungsraumes - nicht überall und um jeden Preis erreicht werden können. Dies wäre wiederum das Opfer, das der bundestaatliche Aufbau, bzw. die Eigenstaatlichkeit der Kantone, fordert.

20 Eine ähnliche Erkenntnis hat übrigens bereits die Europäische Union angeleitet, für die sich die Schweiz gerne als Vorbild sieht - von der sie sich aber im Gegenzug und wider gängiger Abwehrreflexe auch inspirieren lassen darf. Artikel 293 des Römer Vertrages (in der Fassung von Amsterdam) verwies noch bezüglich gewisser Sachbereiche auf mitgliedstaatliche Verhandlungen mit dem Ziel, zumindest soweit als nötig internationale Abkommen zu schliessen (etwa bezüglich der direkten Besteuerung oder der Anerkennung juristischer Personen). Doch mit dem Vertrag von Lissabon wurde dieser Artikel ohne Weiteres gestrichen. ${ }^{19}$

$19 \overline{\text { Für mehr Details, siehe meinen Aufsatz: Thoma }}$ Burri, «Free Movement of Algorithms - Artifici-
Einstimmigkeitserfordernis und die Möglichkeit differenzierter Integration innerhalb der Union wahren die Eigenständigkeit der Mitgliedstaaten zur Genüge. Zwielichtige internationale $\mathrm{Ab}$ kommen zwischen den Mitgliedstaaten in Kompetenzbereichen, die der Union nahe sind und in denen eine «Koordination» notwendig ist, die Mitgliedstaaten aber höchst sensitiv sind, werfen mehr Fragen auf, als sie Antworten geben. Es erweist sich als künstlich, diese Bereiche von den Unionsinstitutionen fernzuhalten. Das Risiko bestünde, dass diese Institutionen entwertet oder unproduktiv konkurriert würden. Schliesslich erscheint es auch nicht zwingend notwendig, dass immer alle Mitgliedstaaten überall gleichförmig mitmachen. Diese Erkenntnisse erscheinen ohne Weiteres übertragbar auf die Schweiz, obschon sie ein Bundesstaat ist.

\section{Schluss}

21 Die Perfidie der Bündner Spracheninitiative liegt in ihrer doppelten Wirkung: Einerseits bewirkt sie eine Lockerung der Pflicht der Mehrheit, die Sprache der Minderheit zu lernen; andererseits bewirkt sie eine Verpflichtung der Minderheit(en) auf die Sprache der Mehrheit. Eine solche doppelte Wirkung kann nur in einem Kanton auftreten, in dem es überhaupt eine Minderheit gibt. Im deutschsprachigen Kanton Luzern zum Beispiel ist dies nicht der Fall. ${ }^{20}$ Die zweite Wirkung bleibt dort aus. Die

ally Intelligent Persons Conquer the European Union's Internal Market», in Woodrow Barfield und Ugo Pagallo (Hrsg.), Research Handbook on the Law of Artificial Intelligence, Edward Elgar, im Erscheinen - im Moment auf SSRN.com, insbesondere Fn. 41 für weitere Verweise.

20 Zur Luzerner Spracheninitiative vom 17. September 2014, Lienhard / Nuspliger (Fn. 10), S. 116 ff. 
gleichlautende Luzerner Spracheninitiative ist deswegen unverfänglicher und moralisch wesentlich weniger problematisch als die Bündner Spracheninitiative. In Luzern erscheint die Initiative bloss als ein unfreundlicher Akt, der Wirkungen einzig gegenüber den anderen Kantonen hat und die Stellung der französischen Sprache in Frage stellt. Doch die Romands sind in der Schweiz stark und robust. Ein Akt der Unfreundlichkeit geht an ihnen spurlos vorüber. Auch der schweizerische Bundesstaat ist stark genug, um mit der ausschliesslich kantonsübergreifenden Wirkung der Luzerner Spracheninitiative zu leben. Echter, gelebter Föderalismus hat eben auch seinen Preis.

Im Kanton Graubünden sieht es jedoch anders aus. Dort entfaltet die Spracheninitiative ihre volle, doppelte Wirkung innerhalb des Kantons und damit ihre ganze Perfidie. Während im Graubünden bloss die erste Wirkung «zugunsten» der deutschsprachigen Mehrheit als ein Akt der Unfreundlichkeit erscheint, ist die zweite Wirkung, jene zuungunsten der Rätoromanen und der Italienischsprachigen, ein echter Akt der Unterdrückung. Die zweite Wirkung macht deswegen die Bündner Spracheninitiative höchst unmoralisch. Die im Vorfeld des Entscheides der Bündner Regierung über die Ungültigkeit der Initiative involvierten Gutachter, Adriano Previtali und Bernhard Ehrenzeller, ${ }^{21}$ empfahlen daher den rechtlichen Spielraum voll auszuschöpfen und die Bündner Spracheninitiative ungültig zu erklären. Doch im

21 Previtali, «Nur eine Fremdsprache in der Primarschule? Ein Rechtsgutachten (Übersetzung)» und Gutachten zur Frage der Gültigkeit der kantonalen Volksinitiative «Nur eine Fremdsprache in der Primarschule» (Fn. 10).
Gegensatz zur Regierung konnten die Gerichte von der ausschöpfenden Interpretation der Verfassungen und der interkantonalen Vereinbarungen nicht überzeugt werden.

23 Der Entscheid des Bundesgerichts kam letztlich wenig überraschend, denn die rechtlichen Grundlagen sind schwach. Die Bundesrechtsbestimmungen sind minimal und decken ungleiche Situationen, wie jene im Kanton Luzern und jene im Kanton Graubünden, mit gleichen Regeln ab. Die zweite Wirkung im Kanton Graubünden (jene zuungunsten der rätoromanisch- und italienischsprachigen Minderheiten) findet im Bundesrecht kaum Resonanzraum. Das allgemeine Diskriminierungsverbot und das Rechtsgleichheitsgebot in Artikel 8 der Bundesverfassung erweisen sich als zu schwach, um die Wirkung aufzufangen. ${ }^{22}$ Die Sprachenartikel wiederum sind lediglich Kompetenzartikel, die eine Widerrechtlichkeit der Bündner Spracheninitiative eben so wenig zu tragen vermögen wie die unglücklichen interkantonalen Vereinbarungen, deren Verbindlichkeit ohne Intervention des Bundes auf den nicht partizipierenden Kanton Graubünden nicht erstreckbar ist. ${ }^{23}$

22 Urteil des Bundesgerichts 1C_267/2016 vom 3. Mai 2017, Erw. 5.

23 Waldmann (Fn. 10), S. 15, zieht die Schlussfolge: «Vor diesem Hintergrund ist es nicht zulässig, kantonale Volksinitiativen, die einen Austritt aus dem HarmoS-Konkordat verlangen oder den Fremdsprachenunterricht auf Primarschulstufe auf eine Fremdsprache begrenzen wollen, wegen Art. 62 Abs. 4 BV für ungültig zu erklären. Einzig Initiativen, die der programmatischen Zielbestimmung von Art. 15 Abs. 3 Sp[rachen]G[esetz] (Kompetenzen in mindestens einer zweiten Landessprache und einer weiteren Fremdsprache am Ende der obligatorischen Schulzeit) widersprechen, dürften wegen Verletzung der bundesstaatlichen Treuepflicht nicht zur Volksabstimmung zugelassen werden.» 
24 Minderheiten sind in der Schweiz exponiert und werden vom Verfassungsrecht kaum explizit geschützt. Eine wohlwollende Leseart davon wäre nun, dass der Minderheitenschutz irgendwo im Durcheinander des Föderalismus, sozusagen zwischen den Kompetenzen des Bundes und des Kantons, verloren gegangen ist. Ähnlich wie beim Doppel im Tennis, wo manchmal beide Partner denken, der jeweils Andere würde einen durch die Mitte geschlagenen Ball schon spielen, ${ }^{24}$ vertrauen sowohl Bund wie Kantone darauf, dass der Minderheitenschutz Sache des Anderen ist. Eine realistischere Leseart hingegen erkennt eine Form der Unterdrückung. Der mangelnde Schutz der Minderheiten erlaubt es der deutschsprachigen Mehrheit im Ernstfall, ihre eigenen Interessen in den Vordergrund zu stellen - auf Kosten der sprachlichen Minderheiten.

24 David Foster Wallace, Infinite Jest, Backbay, Boston, 1996, S. 381 (2006 Jubiläumsausgabe): «[T]he Dems and G.O.P.s stood on either side watching dumbly, like doubles partners who each think the other's surely got it». 